\title{
LA ASOCIACIÓN INTERNACIONAL DE LA SEGURIDAD SOCIAL Y LA SEGURIDAD Y SALUD EN EL TRABAJO
}

\author{
HANS-HORST KONKOLEWSKY
}

\author{
Secretario General \\ Asociación Internacional de la Seguridad Social
}

\begin{abstract}
La Asociación Internacional de la Seguridad Social (AISS) es la organización líder mundial que reúne a las administraciones y a las instituciones nacionales de seguridad social. En su calidad de voz independiente de la seguridad social, la AISS brinda asistencia a sus miembros y responsables de las decisiones en materia de politicas para hacer frente a los retos y al desarrollo de los sistemas de seguridad social, mediante plataformas de cooperación e investigación, producción y transferencia de conocimientos, y la promoción de la seguridad social a escala internacional. Creada en 1927, la AISS cuenta hoy con casi 370 organizaciones miembros de más de 150 países. El Secretario General, Hans-Horst Konkolewsky, presenta aquí el punto de vista de la AISS y una reseña de sus actividades en el ámbito de la seguridad y la salud en el trabajo.
\end{abstract}

Desde sus inicios en la década de 1920, la Asociación Internacional de la Seguridad Social (AISS) ha venido destacando la importancia de la prevención como uno de los medios orientados a la mejora de la vida de los trabajadores y de sus familias.

La seguridad social implica más que la simple satisfacción de las necesidades cuando éstas surgen. El concepto de seguridad social incluye, en primer lugar, la prevención de la ocurrencia de los riesgos y la ayuda a los individuos y a las familias para adecuarse lo mejor posible cuando han de afrontarse las contingencias. Esto refleja el vínculo directo y la responsabilidad de la seguridad social en la prevención en todos los niveles.

A pesar de contarse con pruebas claras de que la prevención mantiene a la gente más sana, con toda la ganancia que supone contar con vidas activas más largas, la mayoría de los sistemas nacionales de salud y de los regímenes del seguro de salud se organizan, no para prevenir la enfermedad, sino más bien para tratarla y compensarla después de sobrevenida. En primer término, no cabe duda de que la mayoría de los proveedores de servicios de salud en todo el mundo no estarían de acuerdo en que pudiesen obtenerse ganancias monetarias a la hora de tratar las enfermedades, y mucho menos a la hora de la prevención de la enfermedad. La mayoría de los sistemas de seguridad social están organizados para el pago de prestaciones en caso de accidentes, de enfermedades y de discapacidad, pero no propician que los ciudadanos vivan unas vidas más saludables.

Dejando de lado los argumentos económicos, existen también sólidos argumentos políticos y éticos a favor de la inversión en la prevención, por cuanto sabemos que en muchos países a escala mundial, es grande el número de ciudadanos que nunca llegan al momento de tener que consumir una asistencia médica cara, debido a que fallecen mucho antes. Existen nuevas vías para superar estos desincentivos? Constituyen los costos siempre crecientes de la asistencia médica, sin un fin a la vista de unos medicamentos y unas tecnologías nuevos y caros, un motivo que obliga a los responsables de las políticas a repensar el papel de la prevención en las políticas de seguridad social?

En su Foro de la Seguridad Social celebrado en Moscú en septiembre de 2007, la AISS sugirió un concepto más amplio y dinámico de la seguridad social.

En base a las crecientes evidencias del papel fundamental de la seguridad social en la construcción de unas sociedades más igualitarias, puesto que contribuye a la reducción de la pobreza y es vital para compartir, de una manera más justa, las oportunidades que trae consigo la globalización, el Foro hizo un llamamiento para una mayor voluntad política que se 
encamine a la extensión de la cobertura de la seguridad social, con el fin de hacerla más accesible y sostenible.

Enfoques proactivos y preventivos que pueden apoyar el desarrollo económico y la cohesión social, desempeñan un papel primordial en la nueva visión estratégica de la AISS:

\section{¿QUÉ SIGNIFICA ESTO PARA LA PREVENCIÓN?}

En primer término, la prevención debe ser accesible para todos. De manera especial, los trabajadores de la creciente economía informal, aquellos que trabajan por cuenta propia y los trabajadores de las pequeñas y micro empresas, deberían estar cubiertos y tener el mismo acceso a los servicios de prevención.

En segundo término, la prevención debe ser sostenible. Esto significa que la prevención debe estar incluida como un objetivo estratégico de la empresa y debe aplicarse a través de un enfoque administrativo sistemático en alianza con los trabajadores.

En tercer término, la prevención debe ser proactiva. No basta con prevenir los riesgos tradicionales bien conocidos. En un mundo laboral cambiante, nuevos riesgos emergentes, como los riesgos psicosociales y los riesgos relacionados con las nanotecnologías, una fuerza laboral que envejece y los trabajadores migrantes, requieren ser abordados de modo proactivo.

En cuarto término, la prevención debe ser innovadora. El nuevo mundo laboral conlleva riesgos más complejos para la salud, lo que requiere medidas más innovadoras. El marcado aumento de las enfermedades crónicas, por ejemplo, significa que la prevención en el lugar de trabajo debería ser más abarcadora y no abordar únicamente riesgos estrictamente relacionados con el trabajo, sino que debería comprometerse en actividades de promoción general de la salud.

La seguridad social y la prevención son, más que nunca, necesarias. Ahora bien, a efectos de conseguir su objetivo primordial, la seguridad social debe ser dinámica. Sólo a través del cambio y de la innovación puede la seguridad social responder proactivamente a los desafíos de la globalización y contribuir a la justicia social y a un desarrollo económico más igualitario. La prevención ha estado siempre, y lo estará aún más en el futuro, vinculada con el desarrollo de una seguridad social dinámica.

\section{LA COMISIÓN ESPECIAL SOBRE LA PREVENCIÓN}

El programa de prevención de la AISS comprende dos aspectos diferentes y complementarios. Por una parte, las actividades promocionales de prevención. Por otra parte, las actividades técnicas llevadas a cabo junto con la Comisión Especial sobre la Prevención, de la Asociación, que representa a todos los Comités Internacionales de la AISS (véase más adelante).

Las actividades técnicas comprenden la supervisión de la evolución a escala mundial, así como la realización de encuestas y de estudios sobre áreas problemáticas generales, como el amianto y la prevención en las pequeñas y medianas empresas.

En 2004, en la 28a. Asamblea General de la AISS, celebrada en Beijing, la Comisión Especial adoptó una Declaración contra el amianto, instándose a todos los países productores y exportadores "a prohibir cuanto antes la producción, el comercio y la utilización de todos los tipos de amianto y de los productos que lo contengan" (Anexo I).

A la fecha, sólo 40 países han prohibido este peligroso producto. Como seguimiento de la Declaración, en 2006, la Comisión publicó un folleto informativo, El amianto: hacia una prohibición mundial, en ocho idiomas. La Comisión se encuentra en la actualidad en el proceso de organización de una campaña sobre ese tema, que se desarrollará en el transcurso de los años 2008 a 2010.

Respecto de la prevención en las pequeñas y medianas empresas, se lanzó, el 30 de abril de 2007, una campaña de sensibilización en el Africa de lengua francesa, para llamar la atención sobre los riesgos ocupacionales.

Esta campaña se lleva a cabo conjuntamente con la Asociación Interafricana de Prevención de los Riesgos Ocupacionales (IAPRP) y la Oficina Internacional del Trabajo (OIT).

La Comisión Especial actúa, además, como la institución de coordinación y planificación de una extensa red descentralizada de actividades de la AISS en el área de la prevención, y mantiene una cooperación con otras organizaciones internacionales, como la Comisión de la Unión Europea, la OIT, la Organización Mundial de la Salud y la Comisión Internacional sobre la Salud Ocupacional, por no nombrar sino a algunas de las más importantes. 


\section{COMITESS INTERNACIONALES DE LA AISS PARA LA PREVENCIÓN DE LOS RIESGOS PROFESIONALES}

Los once Comités Internacionales abarcan la prevención de los accidentes en diversos sectores económicos y algunos asuntos transversales:

- Prevención de los Riesgos Profesionales en la Agricultura

- Prevención de los Riesgos Profesionales en la Industria Química

- Prevención de los Riesgos Profesionales en la Construcción y Obras Públicas

- Prevención de los Riesgos Profesionales originados por la Electricidad

- Seguridad de Máquinas

- Prevención de los Riesgos Profesionales en la Industria Metalúrgica

- Prevención de los Riesgos Profesionales en las Minas

- Prevención de los Riesgos Profesionales en los Servicios de Salud

- Investigación en materia de Prevención de los Riesgos Profesionales

- Información en materia de Prevención de los Riesgos Profesionales

- Educación y Formación en materia de Prevención de Accidentes

Cada Comité Internacional de la AISS actúa como centro de documentación de la información en su ámbito de competencia. Los Comités organizan simposios internacionales, talleres, mesas redondas y reuniones de expertos. En la actualidad, existen más de 25 grupos de trabajo compuestos por expertos internacionales que preparan los futuros simposios internacionales o los folletos técnicos sobre temáticas específicas.

Estas publicaciones técnicas van desde Gas explosions y Back-protecting work practices in health care: Training and prevention concepts in Europe a Work-related health risks: Research and company practice. La mayoría de estos títulos se publican en al menos tres idiomas (ingles, francés y alemán) y algunos en español y en otros idiomas.

Algunos Comités han desarrollado también directrices muy útiles que proporcionan recomendaciones prácticas en su ámbito y que les ha valido una muy buena reputación entre los expertos internacionales.

\section{EL PROTOCOLO DE QUEBBEC}

En 2003, especialmente con la contribución del Instituto Nacional de Seguridad e Higiene en el Trabajo (INSHT) de España, el Comité Internacional para la Educación y Formación desarrolló el Protocolo de la ciudad de Québec para la integración de competencias en salud y seguridad en el trabajo (SST) en la enseñanza y la formación profesionales $y$ técnicas (Anexo II). Este protocolo brinda un marco para la cooperación entre las instituciones que tienen competencias en la prevención de los accidentes laborales y de las enfermedades profesionales, y las instituciones que tienen competencias en educación. En el momento de redactar este documento, aproximadamente diez países se habían comprometido con el protocolo y lo habían firmado.

\section{ESTRATEGIA DE PREVENCIÓN}

El Comité Internacional de la AISS para la Prevención de los Riesgos Profesionales en la Agricultura publicó recientemente el folleto, Seguridad y salud en el Trabajo en las pequeñas explotaciones agrícolas y forestales: Problemas y Soluciones, en diez idiomas. Contiene una estrategia de prevención específica que ha sido desarrollada para las pequeñas empresas agrícolas. La estrategia se orienta hacia las principales áreas seleccionadas:

- La prevención infantil

- La formación básica y el perfeccionamiento

- Las mujeres agricultoras

- Las personas de la tercera edad

- La mano de obra estacional

- Las edificaciones agrícolas

- Las máquinas

- Los trabajos forestales

- La colaboración con asociaciones profesionales

- Los riesgos para la salud en el trabajo

- Los equipos de protección individual

- La legislación

\section{SITIO WEB}

La Comisión Especial y sus once comités internacionales cuentan con un sitio web integral, que brinda todas las informaciones necesarias sobre sus actividades, miembros y publicaciones. Visite:

www.prevention.issa.int 


\section{CONGRESOS MUNDIALES}

Cada tres años, en el marco de las actividades de la Comisión Especial sobre la Prevención, la AISS y la OIT, se organizan conjuntamente los Congresos Mundiales sobre Seguridad y Salud en el Trabajo, con la ayuda material de las organizaciones miembros de la AISS con sede en el país anfitrión. El primero se organizó en Roma, en 1955, y el último, en Florida, en 2005.

Con la participación de 3000 especialistas de todas las regiones del mundo, estos Congresos Mundiales aportan una información actualizada en torno a las nuevas tendencias en seguridad y salud en el trabajo, en educación y formación para la prevención, en cuestiones de medio ambiente, y en soluciones probadas a los muchos problemas vigentes en materia de seguridad y salud en el trabajo. Un objetivo central es la asistencia a los participantes para impedir que tengan que reinventar la rueda, mediante el intercambio de experiencias con sus homólogos de otros países.

El Congreso Mundial marca una fecha esencial en el calendario para todos los responsables de las decisiones y para quienes abordan a diario las cuestiones de prevención, así como para los jefes de las empresas, los directivos gubernamentales, los representantes de las organizaciones de empleadores y de trabajadores, los investigadores, médicos y enfermeras del trabajo, los profesionales de la seguridad, higienistas y especialistas medioambientales, los inspectores de fábricas, administradores de la seguridad social, aseguradores, formadores y educadores, los diseñadores e ingenieros, y todos aquellos interesados en la seguridad, la salud y el medioambiente.

El XVIIII Congreso Mundial sobre Seguridad y Salud en el Trabajo, organizado conjuntamente con la Agencia Coreana para la Seguridad y la Salud en el Trabajo (KOSHA), se celebrará del 29 de junio al 2 de julio de 2008, en Seúl, República de Corea.

El lema del Congreso es "Seguridad y Salud en el Trabajo: Una Responsabilidad de toda la Sociedad", con cuatro temas principales:

- Estrategias y Programas de Seguridad y Salud para el Futuro

- Repercusiones de los Cambios en las Condiciones de Trabajo sobre la Protección del Trabajador

- Nuevos Retos y Oportunidades en el área de la Seguridad y la Salud en el Trabajo
- Sistemas de Gestión de la Seguridad y la Salud en el Trabajo

Por primera vez, una Cumbre sobre Seguridad y Salud se celebrará antes del Congreso Mundial sobre Seguridad y Salud en el Trabajo de 2008. Se invitará a directivos y responsables de decisiones seleccionados, comprometidos en la mejora de la seguridad y la salud en el trabajo, a reflexionar sobre la seguridad y la salud en el trabajo como derecho humano básico y como medio para el crecimiento y el desarrollo económico. La finalidad de la Cumbre será estimular el compromiso político y demostrar que las buenas políticas de seguridad y salud en el trabajo generan una situación de ganancia para los empleadores, los trabajadores y la sociedad en general. Se espera que, como consecuencia, la seguridad y la salud en el trabajo ocupen un lugar más destacado en las agendas nacionales e internacionales.

El Comité Internacional de Prevención de los Riesgos Profesionales originados por la Electricidad y el Comité de Información en materia de Prevención de los Riesgos Profesionales de la AISS, tienen el cometido de organizar un Festival Internacional de Cine y Multimedia que coincida con el Congreso Mundial, ofreciendo a los participantes una visión general de la variedad de películas y productos multimedia disponibles en materia de seguridad y salud en el trabajo. Las discusiones en paneles y los eventos especiales ponen de relieve el papel del video y de los productos multimedia en la prevención. Jurados compuestos por expertos seleccionan los mejores productos y se conceden premios. Los ganadores de los premios de los dos festivales anteriores procedían de Alemania, Canadá, España, India y Reino Unido.

\section{DÍA MUNDIAL DE LA SEGURIDAD Y SALUD EN EL TRABAJO}

Todos los años, la AISS se une al Día Mundial de la Seguridad y Salud en el Trabajo. Celebrado el 28 de abril de cada año, el Día Mundial es una iniciativa de la OIT y se ha venido celebrando desde 2003.

El Día Mundial existe para centrar la atención internacional en el hecho de que la creación y la promoción de una cultura de seguridad y salud pueden contribuir a la reducción, cada año, del número de fallecimientos relacionados con el trabajo.

La AISS apoya plenamente esta iniciativa porque, como se ha visto, la seguridad y la salud en el trabajo se sitúan en el propio centro de nuestra misión de promover la protección de la seguridad social en todo el mundo. 
Desafortunadamente, es frecuente que la seguridad y la salud de los trabajadores sean aún, en muchos países, una preocupación secundaria, en el entendido de que la prevención es demasiado cara. Pero hay claras evidencias de que una buena prevención es un buen negocio, por cuanto aumenta la competitividad, mejora la imagen de la empresa y ejerce un impacto significativo en los costos de la seguridad social.

La AISS opina que la seguridad es responsabilidad de todos los interesados, puesto que unos indivi- duos sanos constituyen la base de la productividad y de unas actividades eficaces en función de los costos. Un objetivo clave de la seguridad social debería ser, en consecuencia, la preservación de la salud de todos. Si se considera a escala humana en términos éticos simples o a través de una perspectiva multinacional, es interés de todos la protección a largo plazo del bienestar de los trabajadores, en aras de la creación de entornos laborales más seguros y de la prevención de accidentes y de su costo humano. En otras palabras, se trata de asumir las responsabilidades.

\section{ANEXO I \\ Declaración contra el amianto}

Beijing, 2004

La Comisión Especial sobre la Prevención de la Asociación Internacional de la Seguridad Social (AISS), reunida en Beijing el 16 de septiembre de 2004, durante la 28 ${ }^{\mathrm{a}}$ Asamblea General de la Asociación, adoptó la siguiente declaración, dirigida a los países que todavía producen o utilizan amianto:

- El amianto es un mineral natural. Según el conjunto de datos epidemiológicos mundiales, el polvo de las fibras que se crea durante la extracción, transformación y utilización de todas las formas de amianto - incluido el crisolito - es cancerígeno para el hombre. Las estadísticas de enfermedades provocadas por el amianto (asbestosis, cáncer de pulmón y de laringe, mesotelioma) permiten estimar en varios cientos de miles el número de personas afectadas cada año en todo el mundo por una infección debida a una exposición profesional al amianto, y en varios miles el número de personas que fallecen cada año a consecuencia de estas enfermedades.

- El amianto se utilizó durante todo el siglo XX para fabricar productos muy diversos. Independientemente de las transformaciones a que se someta este material, sus propiedades peligrosas siguen latentes.

- Ya se han pagado varios miles de millones de dólares estadounidenses en concepto de indemnización, y numerosas empresas han quebrado al no poder seguir haciendo frente a las demandas de indemnización.

- A pesar de las dramáticas consecuencias para la salud humana y de las amenazas que representan para las economías nacionales, cada año se producen y utilizan todavía 2,5 millones de toneladas de amianto.

- Sólo al final de un proceso que ha durado más de treinta años, y tras haber encontrado sustitutos del amianto, algunos países industrializados han decidido prohibir totalmente la producción y utilización del amianto y de productos que lo contengan. En la actualidad, sólo se autorizan en estos países las actividades encaminadas a la eliminación, la descontaminación o el mantenimiento que puedan conllevar un contacto con el amianto.

- Pueden transcurrir varios decenios entre la primera exposición al amianto y la aparición de los primeros indicios patológicos. Todos los países donde no se haya prohibido totalmente este material serán víctimas de los efectos sanitarios y políticos de una auténtica bomba de efectos retardados.

La Comisión Especial sobre la Prevención de la AISS exhorta a todos los países a prohibir cuanto antes la producción, el comercio y la utilización de todos los tipos de amianto y de los productos que lo contengan. 
ANEXO II

Protocolo de la ciudad de Québec para la integración de competencias en salud y seguridad en el trabajo (SST) en la enseñanza y la formación profesionales y técnicas
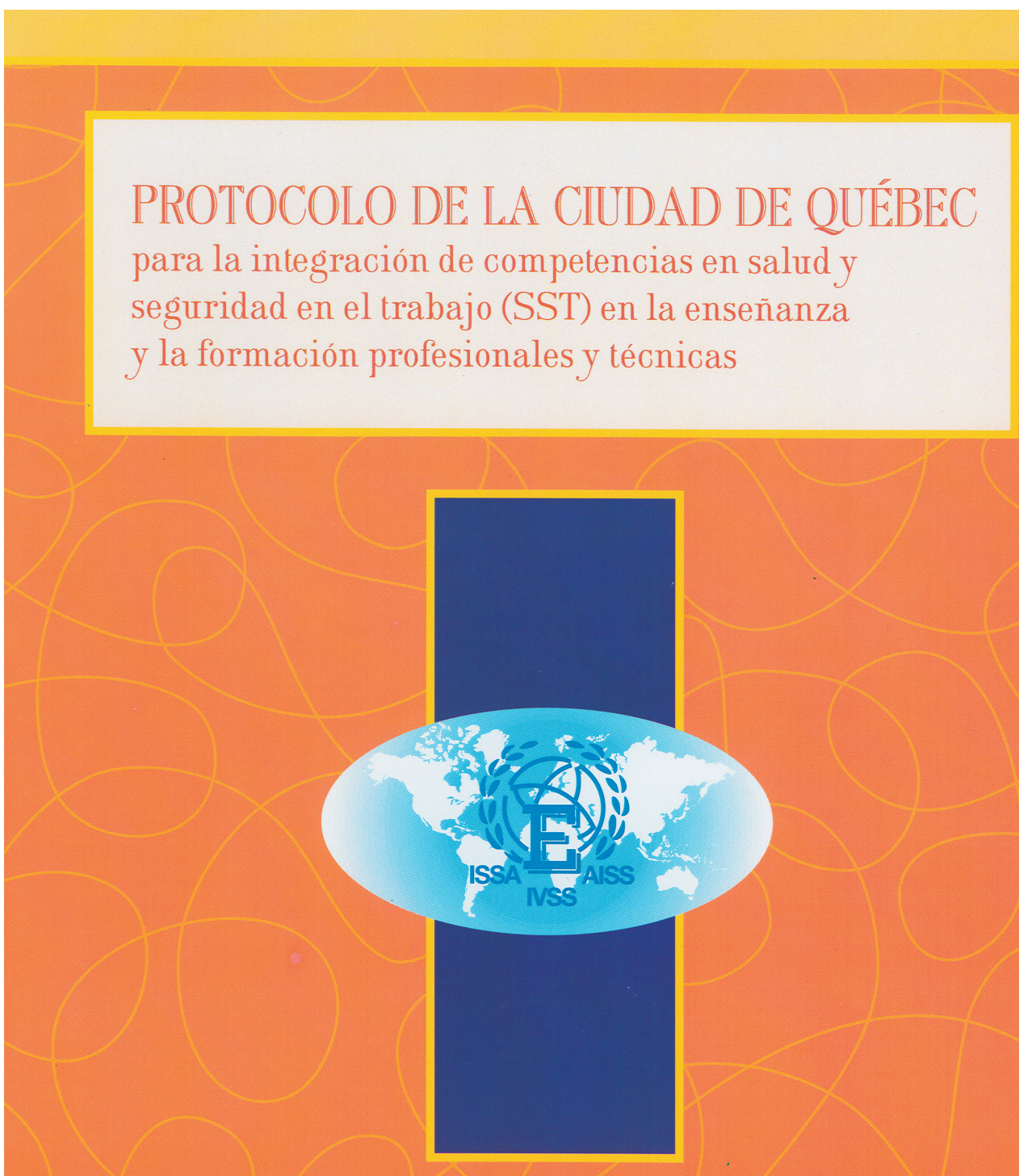


\section{Protocolo de la Ciudad de Québec}

para la integración de competencias en salud y

seguridad en el trabajo (SST) en la enseñanza

y la formación profesionales y técnicas 


\section{Considerando Que:}

- el derecho a la integridad física y psicológica de la persona se ejerce también en el trabajo, desde el inicio del aprendizaje de un oficio,

- el reconocimiento de la salud y la seguridad en el trabajo como valores fundamentales permite incrementar la dimensión social de lo que se ha convenido en llamar globalización,

- la incidencia humana y económica de los accidentes laborales y las enfermedades profesionales es muy elevada en las trabajadoras y los trabajadores jóvenes,

- la capacidad de enfrentar los riesgos profesionales depende en gran medida de la educación recibida en materia de prevención,

- la necesidad de una mejor adecuación entre las realidades del medio laboral y las condiciones del aprendizaje de un oficio es comúnmente reconocida,

el Comité Internacional para la Educación y la Formación en materia de Prevención de la Asociación Internacional de Seguridad Social (AISS), en lo sucesivo llamado "el Comité", propone a los organismos y ministerios nacionales o regionales a cargo de la prevención de los riesgos profesionales y a los de la enseñanza o la formación profesionales y técnicas que adhieran a las recomendaciones del presente Protocolo.

1. Tal como lo afirman organizaciones internacionales como la Organización de las Naciones Unidas (ONU), la Organización de las Naciones Unidas para la Educación, la Ciencia y la Cultura (UNESCO), la Organización Mundial de la Salud (OMS), la Oficina Internacional del Trabajo (OIT) y la Asociación Internacional de Seguridad Social (AISS). 


\section{Preámbulo}

Este Protocolo constituye el marco de referencia para la cooperación entre las instituciones a cargo de la prevención de accidentes laborales y enfermedades profesionales y las instituciones responsables de la educación.

Define los principios y las modalidades de una iniciativa concreta que integra la salud y la seguridad en el trabajo (SST) en la enseñanza y la formación profesionales y técnicas y que se traduce en la realización conjunta de actividades.

El presente Protocolo, de alcance internacional, no se propone establecer reglas en materia de prevención de riesgos de accidente y de enfermedades profesionales en el contexto del ejercicio de un oficio, dado que esto incumbe a la legislación vigente en cada país. 


\section{Principios}

Los principios propuestos a las instituciones a cargo de la prevención de accidentes laborales y enfermedades profesionales y las instituciones responsables de la educación para la celebración de sus acuerdos nacionales o regionales con vistas a integrar la SST en la enseñanza y la formación profesionales y técnicas son los siguientes:

I.1. las competencias en materia de SST relacionadas con cada una de las etapas de realización de un trabajo se integran en la formación a medida que se realiza el aprendizaje del oficio,

I.2. el dominio de los conocimientos requeridos y de las prácticas recomendadas en materia de SST es objeto de una evaluación integrada en la formación,

1.3. el ámbito de la formación adopta prácticas ejemplares en materia de salud y seguridad para el estudiante y favorece la aplicación de dichas prácticas mediante políticas o códigos,

I.4. el material, los equipos y el entorno responden a las normas y reglas reconocidas en materia de SST. 


\section{Modalidades}

II.1. Las asociaciones entre prevención y educación se basan en la puesta en común de medios humanos, técnicos y financieros y se desarrollan siguiendo estos ejes:

\section{I1.1.1 determinación de las competencias en SST (conocimientos, habilidades) para:}

- adoptar métodos y técnicas de trabajo seguros,

- identificar las fuentes de peligros, evaluar los riesgos e implantar medios de prevención para eliminarlos o, en su defecto, controlarlos,

- adaptar los comportamientos a los riesgos de las situaciones de trabajo,

- participar en las diferentes estrategias de prevención implementadas,

- permitir que empleadores y trabajadores ejerzan los derechos y asuman las responsabilidades que les corresponden,

II.1.2 elaboración de material didáctico;

II.1.3 formación de docentes de acuerdo a las exigencias de la SST;

II.1.4 intercambio de pericia y experiencia entre los actores de la prevención y de la enseñanza. 


\section{Modalidades}

II.2. La estrecha colaboración entre la educación y la prevención requiere además la participación de las empresas que constituyen el medio laboral, incluso la colaboración de sus diversas formas de organización profesional.

Así:

11.2.1 los organismos con misión educativa deben dar participación tanto a los organismos encargados de la prevención como a las empresas en la definición de los programas de estudios y de formación,

11.2.2 los organismos responsables de la prevención pueden, llegado el caso, apoyar a los establecimientos de enseñanza en sus intentos por eliminar o reducir los riesgos propios en materia de SST,

II.2.3 las empresas, a través de un reconocimiento concreto de las competencias en SST, valorizarán la formación recibida y los comportamientos seguros adquiridos por los jóvenes. 


\section{Adhesión}

Se invita a las instituciones a cargo de la prevención de accidentes laborales y enfermedades profesionales y a las instituciones responsables de la educación a adherir a las recomendaciones del presente Protocolo mediante la presentación ante el Comité de sus acuerdos nacionales o regionales, existentes o venideros, conformes al presente Protocolo. 


\section{Promoción y seguimiento del Protocolo}

El Comité tiene el mandato de velar por el seguimiento y la promoción del presente Protocolo, a saber:

- supervisar el proceso de adhesión al Protocolo;

- promover y apoyar la celebración de acuerdos nacionales o regionales entre las instituciones implicadas;

- verificar que los acuerdos presentados se ajusten a los principios enunciados;

- asistir a las instancias gubernamentales de los diferentes países contribuyendo con su pericia y experiencia en materia de integración de la prevención en la enseñanza;

- preparar, después de tres años, un estado de situación en lo que respecta a la adhesión al presente Protocolo.

La Secretaría General del Comité, depositaria del presente Protocolo y de los acuerdos nacionales o regionales conformes a éste, tiene la misión de elaborar su base de datos y de ponerla a disposición de todas las instancias interesadas para su consulta.

Presentado en la Ciudad de Quebec el 8 de octubre de 2003, con ocasión del II Seminario Internacional sobre Enseñanza en materia de Salud y Seguridad en el Trabajo 
En la elaboración del presente documento participaron miembros de los organismos e instituciones de los siguientes países:

\section{Alemania}

Bundesverband der Unfallkassen (BUK)

Bundesanstalt für Arbeitsschutz und Arbeitsmedizin (BAuA)

Brasil

Fundacentro

Canadá (Québec)

Commission de la santé et de la sécurité du travail du Québec (CSST)

Ministère de l'Éducation du Québec (MEQ)

\section{España}

Instituto Nacional de las Cualificaciones (INCUAL)

Instituto Nacional de Seguridad e Higiene en el Trabajo (INSHT)

\section{Estados Unidos}

National Institute for Occupational Safety and Health (NIOSH)

\section{Francia}

Institut National de Recherche et de Sécurité (INRS)

Ministère de la Jeunesse, de l'Education et de la Recherche

\section{Portugal}

Instituto de Desenvolvimento e Inspecção das Condições de Trabalho (IDICT)

Ministerio da Educação

\section{Reino Unido}

Health and Safety Executive (HSE)

Suiza

Office Fédéral de la Formation professionnelle et de la Technologie (OFPT)

Schweizerische Unfallversicherungsanstalt (SUVA) 